\title{
An orbital period of 0.94 days for the hot-Jupiter planet WASP-18b
}

\author{
Coel Hellier ${ }^{1}$, D. R. Anderson ${ }^{1}$, A. Collier Cameron ${ }^{2}$, M. Gillon ${ }^{3,4}$, L. Hebb ${ }^{2}$, P. F. L. Maxted ${ }^{1}$, D. Queloz ${ }^{3}$, B. Smalley ${ }^{1}$, \\ A. H. M. J. Triaud ${ }^{3}$, R. G. West ${ }^{5}$, D. M. Wilson ${ }^{1}$, S. J. Bentley ${ }^{1}$, B. Enoch ${ }^{2}$, K. Horne ${ }^{2}$, J. Irwin ${ }^{6}$, T. A. Lister ${ }^{7}$, M. Mayor ${ }^{3}$, \\ N. Parley ${ }^{2}$, F. Pepe ${ }^{3}$, D. L. Pollacco ${ }^{8}$, D. Segransan ${ }^{3}$, S. Udry ${ }^{3}$ \& P. J. Wheatley ${ }^{9}$
}

The 'hot Jupiters' that abound in lists of known extrasolar planets are thought to have formed far from their host stars, but migrate inwards through interactions with the proto-planetary disk from which they were born ${ }^{1,2}$, or by an alternative mechanism such as planet-planet scattering ${ }^{3}$. The hot Jupiters closest to their parent stars, at orbital distances of only $\sim 0.02$ astronomical units, have strong tidal interactions ${ }^{4,5}$, and systems such as OGLE-TR-56 have been suggested as tests of tidal dissipation theory ${ }^{6,7}$. Here we report the discovery of planet WASP-18b with an orbital period of 0.94 days and a mass of ten Jupiter masses $\left(10 M_{\mathrm{Jup}}\right)$, resulting in a tidal interaction an order of magnitude stronger than that of planet OGLE-TR-56b. Under the assumption that the tidaldissipation parameter $Q$ of the host star is of the order of $10^{6}$, as measured for Solar System bodies and binary stars and as often applied to extrasolar planets, WASP-18b will be spiralling inwards on a timescale less than a thousandth that of the lifetime of its host star. Therefore either WASP-18 is in a rare, exceptionally shortlived state, or the tidal dissipation in this system (and possibly other hot-Jupiter systems) must be much weaker than in the Solar System.

Through monitoring by the WASP-South transit survey ${ }^{8}$, coupled with radial-velocity observations from the Coralie spectrograph, we have discovered a $10-M_{\text {Jup }}$ planet transiting the star WASP-18 (= HD 10069) every 0.94 days (Fig. 1). WASP-18b is the first confirmed hot-Jupiter planet that has a period of less than one day (candidates with periods of less than a day have previously been announced based on photometry alone ${ }^{9}$, though experience shows that less than $10 \%$ of such candidates are actual planets ${ }^{10}$ ).

From comparison of the host star to stellar evolutionary tracks ${ }^{11,12}$ (see the Supplementary Information) we find a stellar mass of $1.24 \pm 0.04$ solar masses, $M_{\odot}$, and an age of $630_{-530}^{+950} \mathrm{Myr}$, which is short compared to the approximately 5-Gyr main-sequence lifetime of a star of this mass. A further age constraint is that the observed lithium abundance of WASP-18 is below that typical of F6 stars in the Pleiades (age $120 \mathrm{Myr}$ ) but comparable to that in the Hyades ${ }^{13}$ (age $600 \mathrm{Myr}$ ). Thus we conclude that WASP-18 has an age of $0.5-1.5 \mathrm{Gyr}$, making it one of the youngest known planet-hosting stars.

The theory of tidal interaction for hot Jupiters in close orbits ${ }^{4,5,14}$ predicts that the tidal bulge on the star, raised by the planet, exerts a torque that drains angular momentum from the planet's orbit, causing it to spiral inwards (this arises when the planetary orbit is shorter than the stellar rotation, and contrasts with the Earth-Moon system where the longer orbit of the Moon compared to Earth's spin causes it to move away over time). The spiral infall timescale is determined by the mass and orbital distance of the planet, and by the tidal dissipation parameter of the host star, $Q$. This quality factor is the ratio of the
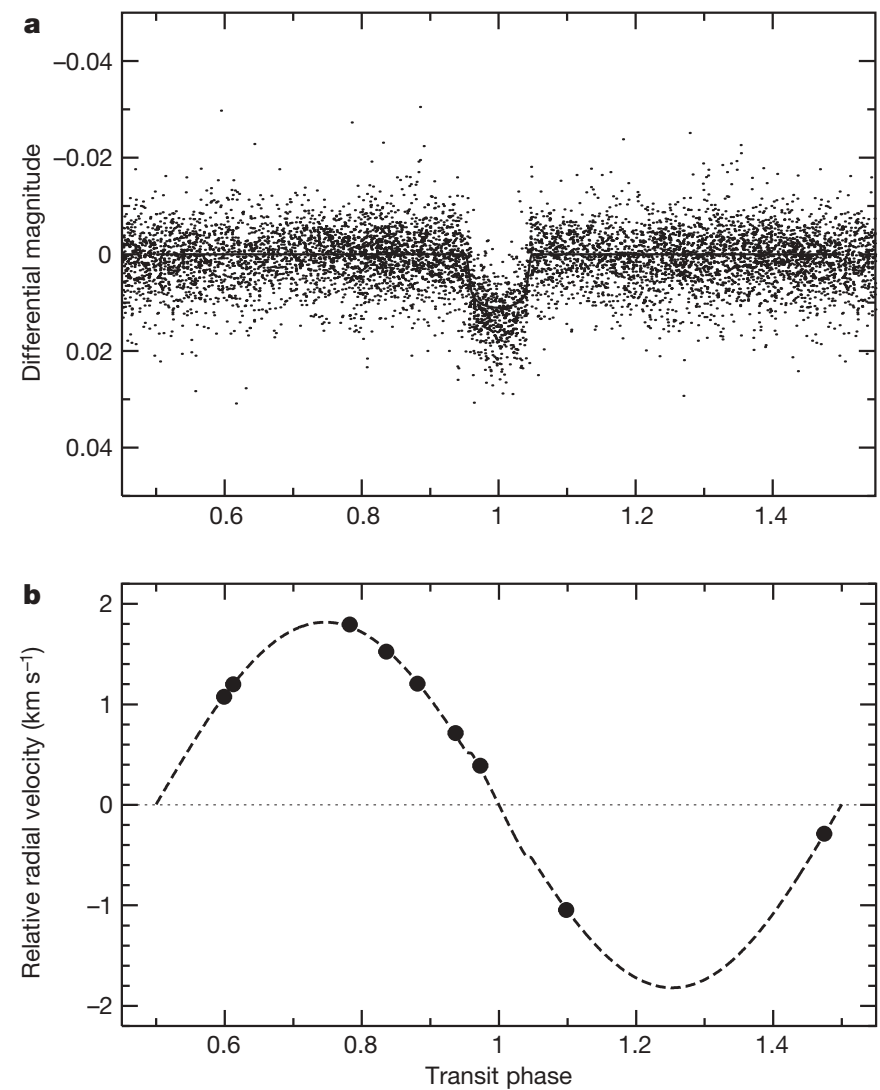

Figure 1 | Discovery data for WASP-18b. a, The WASP-South lightcurve folded on the 0.94-day transit period, together with the model curve from the parameters of Table 1. Monitoring from May-December in 2006 and 2007 resulted in 8,235 photometric data points. $\mathbf{b}$, Coralie radial-velocity measurements, again with the best-fitting model. The parameters of the system, derived from ${ }^{26}$ the radial-velocity data, the WASP photometry, and additional transit photometry from the Euler telescope, are given in Table 1. The parameters of the host star in Table 2 were derived independently ${ }^{27}$ from the stellar spectra. The stellar rotation rate $(v \sin i)$ is $11.0 \pm 1.5 \mathrm{~km} \mathrm{~s}^{-1}$, which (assuming that the spin and orbit are aligned) implies a rotation period of 5.6 days, typical for a young $\mathrm{F}$ star.

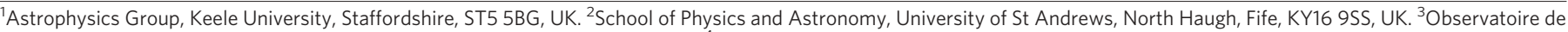

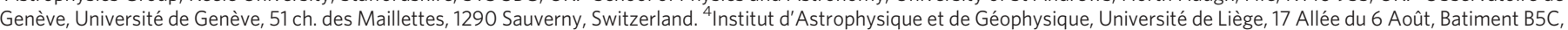

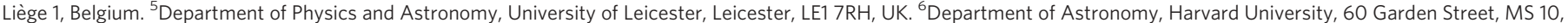

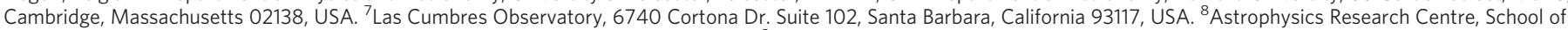
Mathematics and Physics, Queen's University, University Road, Belfast, BT7 1NN, UK. 'Department of Physics, University of Warwick, Coventry, CV4 7AL, UK. 
Table 1 | System parameters for WASP-18

\begin{tabular}{|c|c|}
\hline Parameter & Value \\
\hline $\begin{array}{l}\text { Transit epoch, } T_{\mathrm{C}} \\
\text { Orbital period, } P \\
\text { Transit duration, } T_{14} \\
\text { Planet/star area ratio, } R_{\mathrm{P}}^{2} / R_{*}^{2} \\
\text { Impact parameter, } b=a \cos i / R_{*} \\
\text { Stellar reflex velocity, } K_{1} \\
\text { Centre-of-mass velocity, } \gamma \\
\text { Orbital semi-major axis, } a \\
\text { Orbital inclination, } i \\
\text { Orbital eccentricity, e } \\
\text { Argument of periastron, } \omega \\
\text { Stellar mass, } M_{*} \\
\text { Stellar radius, } R_{*} \\
\text { Stellar surface gravity, log } g_{*} \\
\text { Stellar density, } \rho_{*} \\
\text { Planet mass, } M_{\mathrm{P}} \\
\text { Planet radius, } R_{\mathrm{P}} \\
\text { Planet surface gravity, log } g_{\mathrm{P}} \\
\text { Planet density, } \rho_{\mathrm{P}} \\
\text { Planet surface temperature, } T_{\mathrm{P}}\end{array}$ & $\begin{array}{l}\text { BJD } 2,454,221.48163 \pm 0.00038 \\
0.94145299 \pm 0.00000087 \text { days } \\
0.08932 \pm 0.00068 \text { days } \\
0.00875 \pm 0.00021 \\
0.25 \pm 0.15 \\
1.8183 \pm 0.0080 \mathrm{~km} \mathrm{~s}^{-1} \\
3.1961 \pm 0.0033 \mathrm{~km} \mathrm{~s}^{-1} \\
0.02026 \pm 0.00068 \mathrm{AU} \\
86.0 \pm 2.5^{\circ} \\
0.0092 \pm 0.0028 \\
-96 \pm 10^{\circ} \\
1.25 \pm 0.13 \mathrm{M}_{\odot} \\
1.216_{-0.054}^{+0.067} R_{\odot} \\
4.367_{-0.042}^{+0.028}(\mathrm{cgs}) \\
0.707_{-0.096}^{+0.056} \rho_{\odot} \\
10.30 \pm 0.690_{\text {Jup }} \\
1.106_{-0.0054}^{+0.072} R_{\text {Jup }} \\
4.289_{-0.050}^{+0.027}(\mathrm{cgs}) \\
7.73_{-1.27}^{+0.78} \rho_{\text {Jup }} \\
2,384_{-30}^{+58} \mathrm{~K}\end{array}$ \\
\hline
\end{tabular}

$\overline{B J D}$, barycentric Julian day. Subscripts $P, \odot$ * and Jup refer to planetary, solar, stellar and Jupiter values, respectively. To convert cgs units to SI units for the gravity values, subtract two.

available energy to the amount dissipated by frictional losses during each orbital forcing cycle.

$Q$ is found to be of the order of $10^{5}$ to $10^{6}$ from studies of binary stars ${ }^{15}$ and the gas-giant planets in our Solar System ${ }^{16,17}$ (in which it is often supposed that the $Q$ value of stars and gas-giant planets will be similar). Thus values of $Q=10^{5}-10^{6}$ are often applied to the starplanet tides of hot Jupiters ${ }^{18-20}$. However, for $Q \approx 10^{6}$ the future lifetime of WASP-18b is only $0.65 \mathrm{Myr}$ (Fig. 2), which is $10^{-4}$ of the estimated lifetime $(\sim 5 \mathrm{Gyr})$ of the host star WASP-18. Thus, either WASP-18 is in an exceptionally shortlived state, or its $Q$ value is much higher. Matching the infall timescale to the current age of WASP-18 gives a $Q$ as high as $10^{9}$, in line with some previous indications $s^{7,21,22}$.

For comparison, WASP-18b's infall timescale is an order of magnitude shorter than that of the much-discussed OGLE-TR-56b $\mathrm{b}^{6,7}$ (assuming that $Q$ is the same for both), and gives a current rate of period change of $-0.00073\left(10^{6} / Q\right) \mathrm{s} \mathrm{yr}^{-1}$. For low values of $Q$ this would accumulate to a detectable change in transit epoch in less than a decade (for $Q=10^{6}$ the transit time shifts by $28 \mathrm{~s}$ after $10 \mathrm{yr}$, which compares with a currently achievable timing accuracy ${ }^{23}$ of $5 \mathrm{~s}$ ). Thus WASP-18b is a diagnostic planet, either (for a low Q) being an exceptionally rare object in which the tidal decay is directly measurable, or forcing a reappraisal to much higher $Q$ values; either way it will help establish the dynamical ages of the class of hot-Jupiter planets. WASP18 will also help constrain our understanding of stellar interiors, given that the $Q$ value depends on the dissipation of interior waves excited by the tidal forcing ${ }^{7}$.

For all values of $Q$ up to about $10^{10}$ the planet will spiral inwards to destruction within the star's main-sequence lifetime. The trajectories in Fig. 2 continue until the planet reaches its Roche limit, at which

Table 2 | Stellar parameters of WASP-18

\begin{tabular}{ll}
\hline Parameter & Value \\
\hline Position, J2000 & $01 \mathrm{~h} 37 \mathrm{~min} 25.03 \mathrm{~s}$, \\
Brightness, V mag & $-45^{\circ} 40^{\prime} 40.3^{\prime \prime}$ \\
Spectral type & 9.3 \\
Parallax & $\mathrm{F} 6$ \\
Distance & $10.06 \pm 1.07 \mathrm{mas}$ (ref. 25) \\
Temperature, $T_{\text {eff }}$ & $100 \pm 10 \mathrm{pc}$ \\
Gravity, logg & $6,400 \pm 100 \mathrm{~K}$ \\
Turbulence, $\xi_{\mathrm{t}}$ & $4.4 \pm 0.15(\mathrm{cgs})$ \\
Rotation, vsini & $1.6 \pm 0.1 \mathrm{~km} \mathrm{~s}^{-1}$ \\
Metallicity, $[\mathrm{Fe} / \mathrm{H}]$ & $11.0 \pm 1.5 \mathrm{~km} \mathrm{~s}^{-1}$ \\
Lithium, logN(Li) & $0.00 \pm 0.09$ \\
Age & $2.65 \pm 0.08$ \\
\hline
\end{tabular}

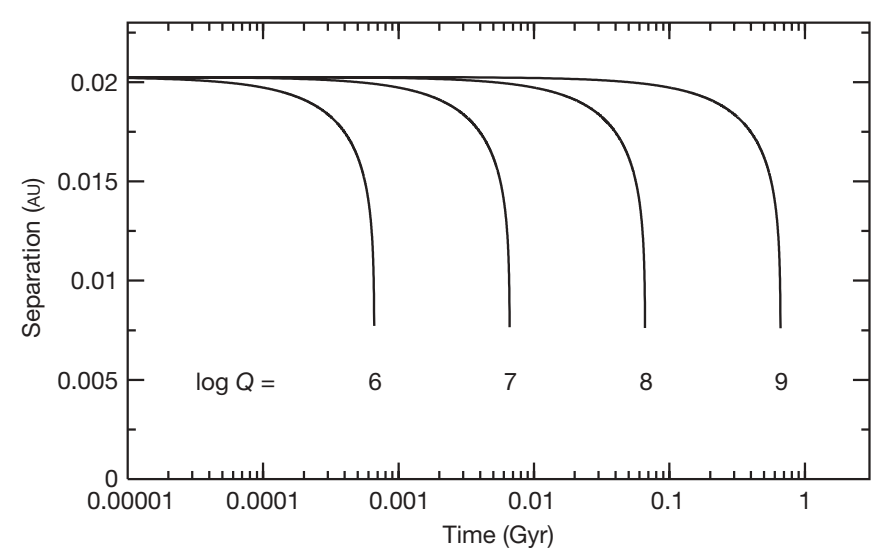

Figure 2 | Future evolution of WASP-18b. The decrease in the orbital semimajor axis for different values of the tidal quality factor $Q$. The spiral infall timescale is given by (see equation (29) of ref. 14 and equation (5) of ref. 18):

$$
t_{\text {remain }} \approx \frac{2 Q}{117 n}\left(\frac{M_{\mathrm{s}}}{M_{\mathrm{p}}}\right)\left(\frac{a}{R_{\mathrm{s}}}\right)^{5}
$$

where $n$ is the orbital angular frequency, $M_{\mathrm{s}} / M_{\mathrm{p}}$ is the ratio of stellar to planet masses, $a / R_{\mathrm{s}}$ is the ratio of the orbital semi-major axis to the stellar radius, and the star is assumed to rotate slowly. The $Q$ parameter measures the inverse fraction of the available energy dissipated in the star by frictional processes per tidal forcing cycle. It is usually expressed as a dimensionless ratio of the tidal quality factor of the star to the tidal Love number ${ }^{14,19}$.

point it will be tidally disrupted, and its material will likely feed onto the star through the Lagrangian point (this ignores radiative evaporation, which will be hindered by the relatively high surface gravity of this massive planet). Assuming that the angular momentum is assimilated by the star, it would be spun up from a rotation rate of 5.6 days to about 0.7 days, and thus be reborn as a rapidly rotating star. Heavier elements from the planetary core could contaminate the stellar atmosphere, where, owing to the relatively small convection layer of an F6 star, they might be readily visible. The planet-hosting star HD 82943 shows evidence of planet engulfment ${ }^{24}$, although it is currently rotating slowly, and thus any engulfment must have occurred long enough ago for magnetic braking to have since slowed the star.

Received 4 March; accepted 26 June 2009.

1. Lin, D. N. C., Bodenheimer, P. \& Richardson, D. C. Orbital migration of the planetary companion of 51 Pegasi to its present location. Nature 380, 606-607 (1996).

2. Tanaka, H., Takeuchi, T. \& Ward, W. R. Three-dimensional interaction between a planet and an isothermal gaseous disk. I. Corotation and Lindblad torques and planet migration. Astrophys. J. 565, 1257-1274 (2002).

3. Rasio, F. \& Ford, E. B. Dynamical instabilities and the formation of extrasolar planetary systems. Science 274, 954-956 (1996).

4. Hut, P. Tidal evolution in close binary systems. Astron. Astrophys. 99, 126-140 (1981).

5. Eggleton, P. P., Kiseleva, L. G. \& Hut, P. The equilibrium tide model for tidal friction. Astrophys. J. 499, 853-870 (1998)

6. Sasselov, D. D. The new transiting planet OGLE-TR-56b: orbit and atmosphere. Astrophys. J. 596, 1327-1331 (2003).

7. Ogilvie, G. I. \& Lin, D. N. C. Tidal dissipation in rotating solar-type stars. Astrophys. J. 661, 1180-1191 (2007).

8. Pollacco, D. L. et al. The WASP project and the SuperWASP cameras. Publ. Astron. Soc. Pacif. 118, 1407-1418 (2006).

9. Sahu, K. C. et al. Transiting extrasolar planetary candidates in the Galactic bulge. Nature 443, 534-540 (2006).

10. Pont, F. et al. A transiting planet among 23 new near-threshold candidates from the OGLE survey-OGLE-TR-182. Astron. Astrophys. 487, 749-754 (2008).

11. Baraffe, I., Chabrier, G., Allard, F. \& Hauschildt, P. H. Evolutionary models for solar metallicity low-mass stars: mass-magnitude relationships and color-magnitude diagrams. Astron. Astrophys. 337, 403-412 (1998).

12. Hebb, L. et al. WASP-12b: The hottest transiting planet yet discovered. Astrophys. J. 693, 1920-1928 (2009).

13. Boesgaard, A. M. \& Tripicco, M. J. Lithium in the Hyades cluster. Astrophys. J. 302, L49-L53 (1986).

14. Dobbs-Dixon, I., Lin, D. N. C. \& Mardling, R. A. Spin-orbit evolution of short-period planets. Astrophys. J. 610, 464-476 (2004) 
15. Meibom, S. \& Mathieu, R. D. A robust measure of tidal circularization in coeval binary populations: the solar-type spectroscopic binary population in the open cluster M35. Astrophys. J. 620, 970-983 (2005).

16. Goldreich, P. \& Soter, S. Q in the Solar System. Icarus 5, 375-389 (1966).

17. Peale, S. J. Origin and evolution of the natural satellites. Annu. Rev. Astron. Astrophys. 37, 533-602 (1999).

18. Levrard, B. Winisdoerffer, C.\& Chabrier, G. Falling transiting extrasolar giant planets. Astrophys. J. 692, L9-L13 (2009).

19. Jackson, B., Greenberg, R. \& Barnes, R. Tidal heating of extrasolar planets. Astrophys. J. 681, 1631-1638 (2008)

20. Laughlin, G. et al. Rapid heating of the atmosphere of an extrasolar planet. Nature 457, 562-564 (2009)

21. Pätzold, M. \& Rauer, H. Where are the massive close-in extrasolar planets? Astrophys. J. 568, L117-L120 (2002).

22. Pätzold, M., Carone, L. \& Rauer, H. Tidal interactions of close-in extrasolar planets: The OGLE cases. Astron. Astrophys. 427, 1075-1080 (2004).

23. Gillon, M. et al. Improved parameters for the transiting hot Jupiters WASP-4b and WASP-5b. Astron. Astrophys. 496, 259-267 (2009).

24. Israelian, G., Santos, N. C., Mayor, M. \& Rebolo, R. Evidence for planet engulfment by the star HD 82943. Nature 411, 163-166 (2001).

25. van Leeuwen, F. Validation of the new Hipparcos reduction. Astron. Astrophys. $474,653-664$ (2007).
26. Collier-Cameron, A et al. WASP-1b and WASP-2b: two new transiting exoplanets detected with SuperWASP and SOPHIE. Mon. Not. R. Astron. Soc. 375, 951-957 (2007).

27. West, R. et al. The low density transiting exoplanet WASP-15b. Astron. J. 137, 4834-4836 (2009).

Supplementary Information is linked to the online version of the paper at www.nature.com/nature.

Acknowledgements We thank the South African Astronomical Observatory for hosting WASP-South and the UK's Science and Technology Facilities Council for funding.

Author Contributions WASP-S construction, operation and candidate selection (C.H., D.R.A., D.M.W., P.F.L.M., B.S., S.J.B.); WASP-S design (D.L.P); WASP observatory software (J.I., D.R.A., P.F.L.M.); WASP-S data processing (D.R.A., D.M.W., B.S.); WASP data pipeline (A.C.C., T.A.L., N.P., K.H.); transit-search code (A.C.C., L.H., B.E.); WASP data archive (R.G.W., P.J.W.); Coralie/EulerCAM data (M.G., A.H.M.J.T., D.S., D.Q.); Euler/Coralie construction and upgrade (D.Q. M.M., S.U., F.P.); planet characterization (A.C.C., D.R.A., M.G.); host star characterization (B.S., L.H.); paper writing (C.H., A.C.C.).

Author Information Reprints and permissions information is available at www.nature.com/reprints. Correspondence and requests for materials should be addressed to C.H. (ch@astro.keele.ac.uk). 